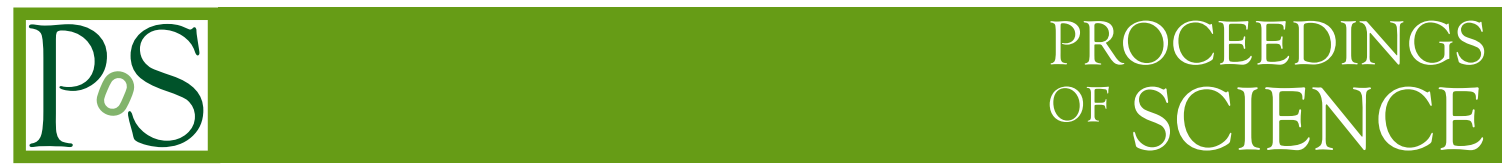

\title{
Searches for invisible Higgs boson decays at the ATLAS experiment
}

\section{Benjamin John Rosser ${ }^{a, *}$ on behalf of the ATLAS collaboration}

${ }^{a}$ Department of Physics and Astronomy, University of Pennsylvania, 209 South 33rd Street, Philadelphia, PA, United States of America

E-mail: bjr@sas.upenn.edu

In the Standard Model, the branching ratio for Higgs boson decays to invisible final states is very small, but it can be significantly enhanced in extensions of the Standard Model. This talk presents searches for Higgs boson decays to invisible final states with the full run 2 data.

40th International Conference on High Energy physics - ICHEP2020

July 28 - August 6, 2020

Prague, Czech Republic (virtual meeting)

${ }^{*}$ Speaker 


\section{Introduction}

Since the discovery of the Higgs boson [1] [2] in 2012, the ATLAS collaboration [3] has been working to study its properties and interactions with other particles. One interesting question is whether the Higgs can decay to an "invisible" final state, that is, neutral particles not directly observable by detectors. In the Standard Model, the only such particle is the neutrino, and the only way a Standard Model Higgs can produce a fully invisible final state is through the $H \rightarrow Z Z^{*} \rightarrow$ $v \bar{v} v \bar{v}$ process, which has the very small calculated branching fraction of $1.05 \times 10^{-3}$ [4]. However, the branching ratio $\mathcal{B}_{H \rightarrow \text { inv }}$ can be significantly enhanced by various Beyond the Standard Model physics. For example, one scenario of particular interest is "Higgs portal" dark matter, in which the Higgs couples directly to a potential dark matter particle. If the dark matter particle has a mass $m_{\mathrm{DM}}<\frac{1}{2} m_{H} \approx 62.5 \mathrm{GeV}$, it should be possible to directly observe this decay at a collider.

Figure 1 shows possible invisible decays in the main four Higgs production modes. To search for one of these signatures, we can look for a large transverse momentum imbalance $\left(E_{\mathrm{T}}^{\text {miss }}\right)$ plus evidence of the production signature in question. The main challenge in doing so is distinguishing these processes from the decay of a $\mathrm{Z}$ boson into neutrinos, which will also produce a large $E_{\mathrm{T}}^{\mathrm{miss}}$ in the final state and may leave a similar signature. To that end, even though the cross section of gluon-gluon fusion (ggF, Figure 1c) is the largest of the four production modes, it is far smaller than that of the $\mathrm{Z}$ decay into neutrinos, and therefore not the most sensitive channel. Instead, the most sensitive is vector boson fusion (VBF, Figure 1a), the second most common production mode, as the VBF Higgs and VBF weak boson cross sections are on the same order of magnitude.

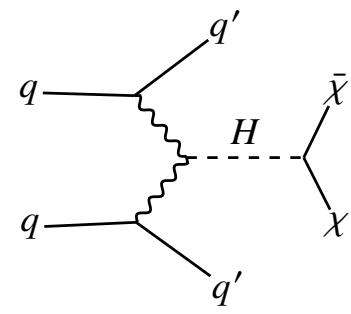

(a) Vector boson fusion (VBF)

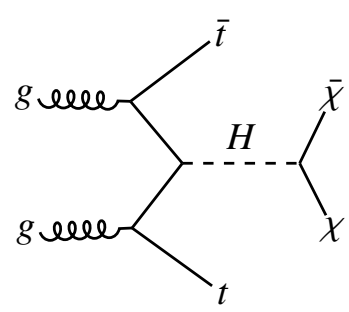

(b) Top fusion (ttH)
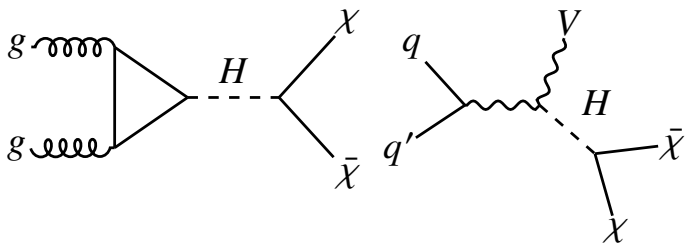

(c) Gluon-gluon fusion (ggF)

(d) Higgs-strahhlung (VH)

Figure 1: Feynman diagrams showing potential invisible Higgs decay in the main Higgs production channels.

ATLAS has performed searches for invisible Higgs decays since run 1; a summary of recent previous analyses containing $36.1 \mathrm{fb}^{-1}$ of $\sqrt{s}=13 \mathrm{TeV}$ proton-proton collision data is shown in Figure 2 [5]. Two new searches with $139 \mathrm{fb}^{-1}$ of $\sqrt{s}=13 \mathrm{TeV}$ data that set limits on $\mathcal{B}_{H \rightarrow \text { inv }}$ have recently been released: an update to the VBF analysis [6] and a new interpretation of the mono-jet analysis [7]. A brief overview of these results is presented in the next two sections.

\section{VBF Higgs to Invisible}

The vector boson fusion $H \rightarrow$ invisible analysis searches for a pair of jets plus a large missing transverse momentum $\left(E_{\mathrm{T}}^{\text {miss }}>200 \mathrm{GeV}\right)$ from the Higgs's decay into invisible particles [6]. The jets from the VBF process tend to be widely separated in pseudorapidity $(\eta)$ with little hadronic 


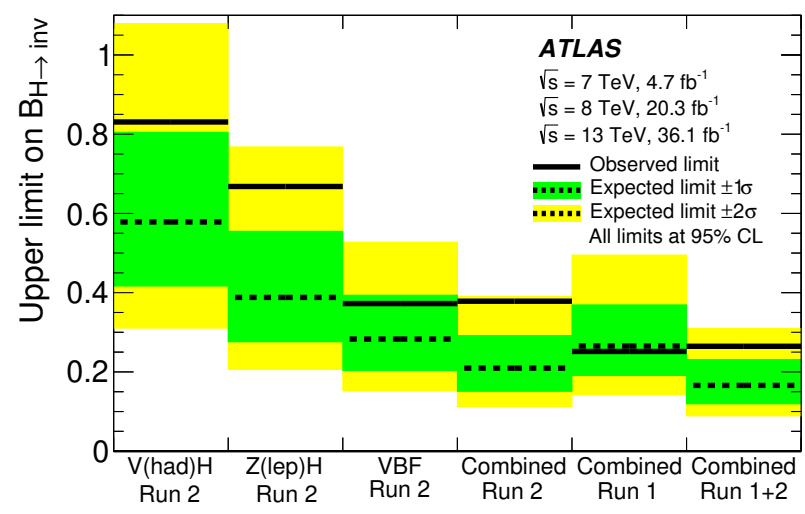

Figure 2: Limits on $\mathcal{B}_{H \rightarrow \text { inv }}$ from the ATLAS collaboration using partial run 2 data $\left(36.1 \mathrm{fb}^{-1}\right)$. Three run 2 analyses are included- two VH searches (V(had) and Z(lep)) and VBF- and are then combined with results from run 1 to set an observed (expected) limit of $0.26\left(0.17_{-0.05}^{+0.07}\right)$. [5]

activity between them. To suppress this background, requirements are imposed on the $\eta\left(\Delta \eta_{j j}>4\right)$ and invariant mass $\left(m_{\mathrm{jj}}>800 \mathrm{GeV}\right)$ of the dijet system, and additional central jets are vetoed. Further, because of the scalar nature of the Higgs, the jets in the final state tend to be more aligned in the transverse plane than the $\mathrm{V}+\mathrm{jets}$ background, so $\Delta \phi_{\mathrm{jj}}$ is required to be less than 2.0.
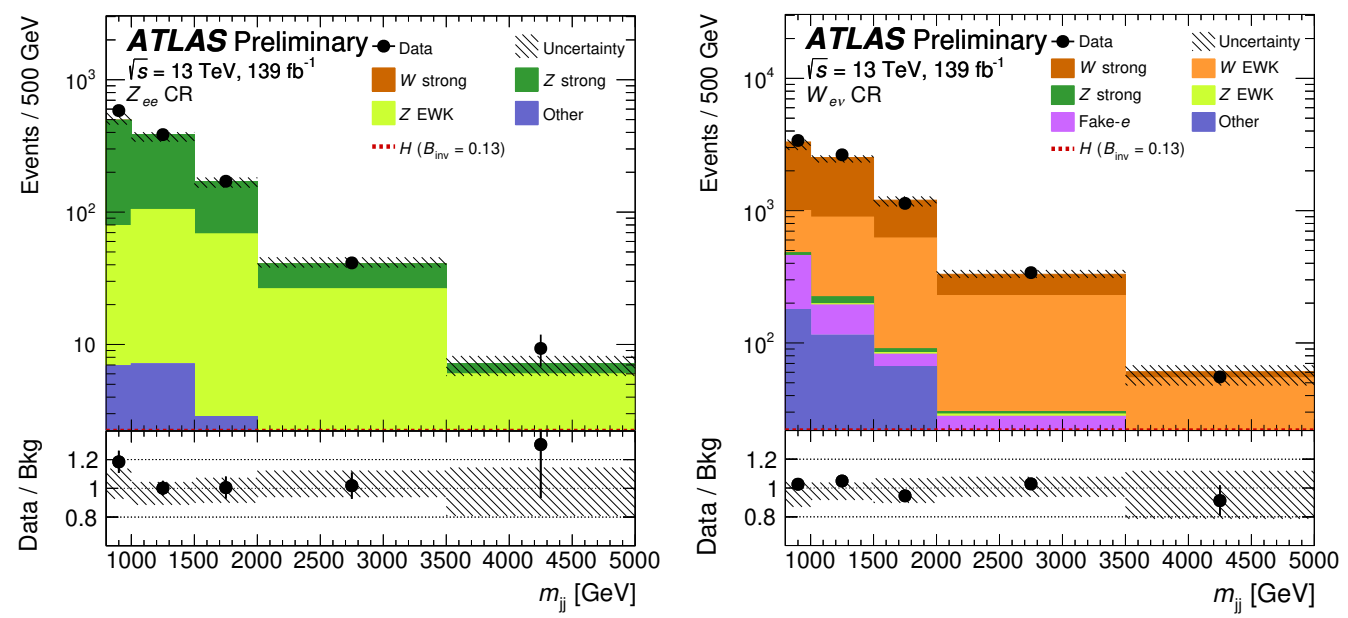

Figure 3: Pre-fit plots of the $Z_{\mathrm{ee}}$ (2-lepton) and $W_{\mathrm{e} v}$ (1-lepton) control region for the VBF analysis. Electroweak-initiated $\mathrm{V}+$ jets processes become the dominant background as $m_{\mathrm{jj}}$ increases. In these regions, the $E_{\mathrm{T}}^{\text {miss }}$ cut is defined using the vector sum of $E_{\mathrm{T}}^{\text {miss }}$ and the transverse momentum ( $\left.p_{\mathrm{T}}\right)$ of the lepton(s). [6]

A partially data driven technique is used to estimate the $Z(v v)+$ jets and $W(l v)+$ jets (with lost lepton) backgrounds. One- and two- lepton control regions are defined, as shown in Figure 3. In these regions, data is compared to a Monte Carlo (MC) simulation of the V+jets processes, and the ratio of data to $\mathrm{MC}$ is taken as a transfer factor to rescale the $\mathrm{MC}$ prediction in the zero-lepton signal region. This process is performed separately for each bin and for $W$ and $Z$ processes: $Z \rightarrow l l$ is used to estimate $Z \rightarrow v v$. The MC used is produced using the SHERPa 2.2 generator [8], with VBFinitiated $\mathrm{V}+$ jets events produced at leading order (LO) and strong-initiated $\mathrm{V}+$ jets events produced 
at next to leading order (NLO). To reduce uncertainties on the background estimate, a parton-level filter was developed in collaboration with the authors of SHERPA to enhance the generated sample size in the high $m_{\mathrm{jj}}$ region.

Two additional backgrounds comes from multijet (QCD) events, in which a jet is either mismeasured as a fake lepton (contaminating the $W$ control regions) or as fake $E_{\mathrm{T}}^{\text {miss }}$ (contaminating the signal region). The fake lepton background is estimated using a dedicated low $E_{\mathrm{T}}^{\text {miss }}$ significance region, and an additional transfer factor is derived for each bin in the $W_{e v}$ control region. The fake $E_{\mathrm{T}}^{\text {miss }}$ background is estimated using a data-driven rebalance and smear technique. A sample of events with no real $E_{\mathrm{T}}^{\mathrm{miss}}$ are extracted from single-jet triggered data, and by smearing the jet $p_{\mathrm{T}}$ multiple times per event, the jet mismeasurement can be simulated to produce a sample of QCD events with fake $E_{\mathrm{T}}^{\text {miss }}$. This multijet estimate is then normalized by a fit to data in $\Delta \phi_{\mathrm{jj}}$, and closure checked in in low- $m_{\mathrm{jj}}$ and low- $E_{\mathrm{T}}^{\text {miss }}$ versions of the signal region.

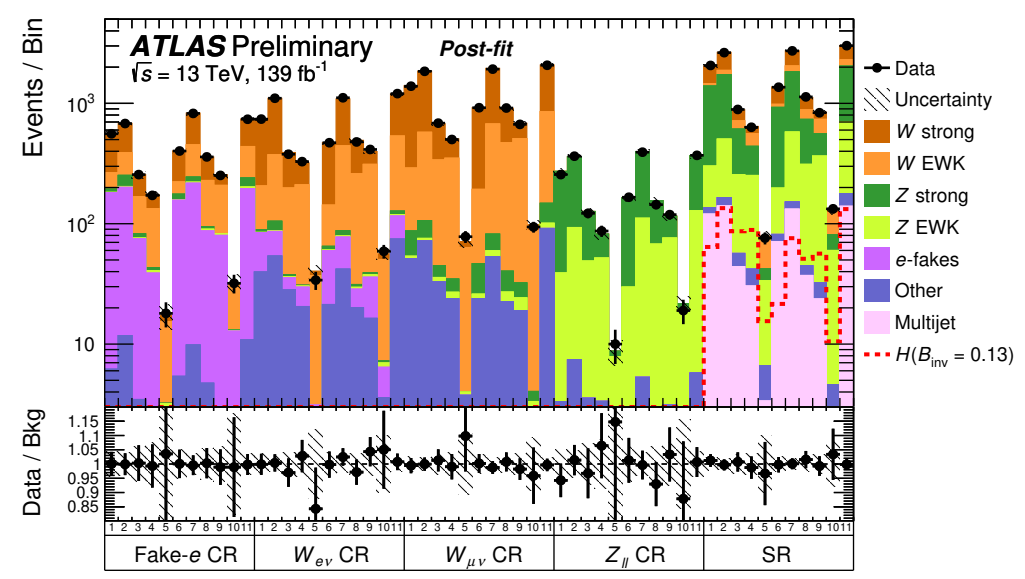

Figure 4: Post-fit plot showing data/MC agreement for all regions and bins in the VBF analysis. The analysis uses a $2 \mathrm{D}$ binning in $\Delta \phi_{j j}$ (bins 1-5 require $\Delta \phi_{j j}<1$ and 6-10 require $1<\Delta \phi_{j j}<2$ ) and $m_{\mathrm{jj}}$. The first ten bins require $N_{\text {jets }}=2$; events with additional forward jets $\left(2<N_{\text {jets }}<4\right)$ are grouped into the 11th bin. [6]

The multijet estimate and transfer factors are applied in a simultaneous likelihood fit across all regions and bins; the results are shown in Figure 4. No significant disagreement between data and Monte Carlo is seen, and so an observed (expected) $95 \%$ confidence limit is set on $\mathcal{B}_{H \rightarrow \text { inv }}$ at $0.13\left(0.13_{-0.04}^{+0.05}\right)$. This is approximately a factor of 3 improvement in sensitivity from the previous version of the VBF analysis (shown in Figure 2), where the observed (expected) limit was 0.37 $\left(0.28_{-0.08}^{+0.11}\right)$. The largest source of uncertainty on this limit is data statistics, followed by V+jets simulation statistics and multijet background estimation.

This result can also be interpreted as a limit on the decay of the Higgs into dark matter particles, such as a weakly interacting massive particle (WIMP) [9]. Figure 5 shows that results from this analysis are quite complementary to limits set by direct dark matter detection experiments.

\section{Monojet}

The mono-jet analysis searches for a large missing transverse momentum $\left(E_{\mathrm{T}}^{\mathrm{miss}}>200 \mathrm{GeV}\right)$, plus at least one additional jet, which is required to have $p_{T}>150 \mathrm{GeV}$ and $|\eta|<2.4$. This search 


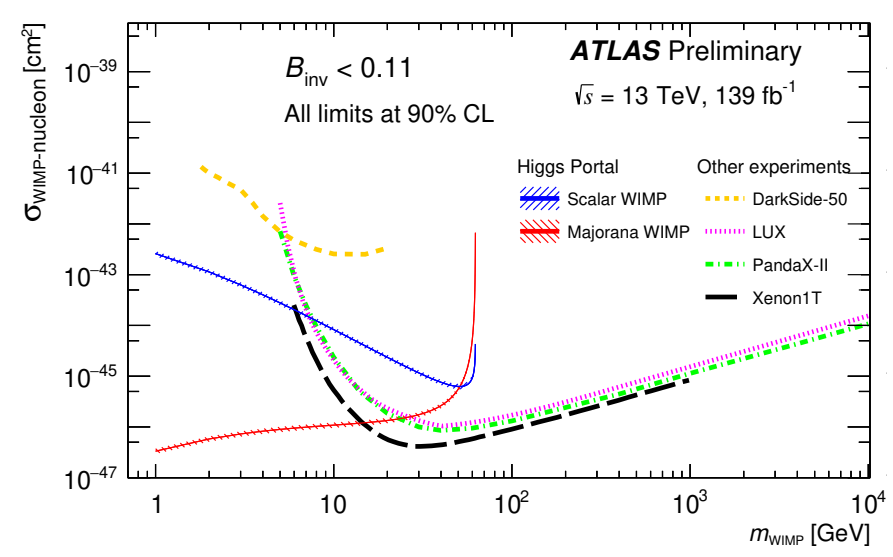

Figure 5: $\mathcal{B}_{H \rightarrow \text { inv }}$ limit from VBF analysis interpreted as a limit on the spin-independent WIMP-nucleon elastic scattering cross section for two Higgs portal models in which the Higgs decays to WIMPs. These limits are then compared to results from direct detection experiments. [6]

has many possible interpretations, one of which is gluon-gluon fusion $H \rightarrow$ invisible in which a jet is produced as initial state radiation from the production of the Higgs [7].
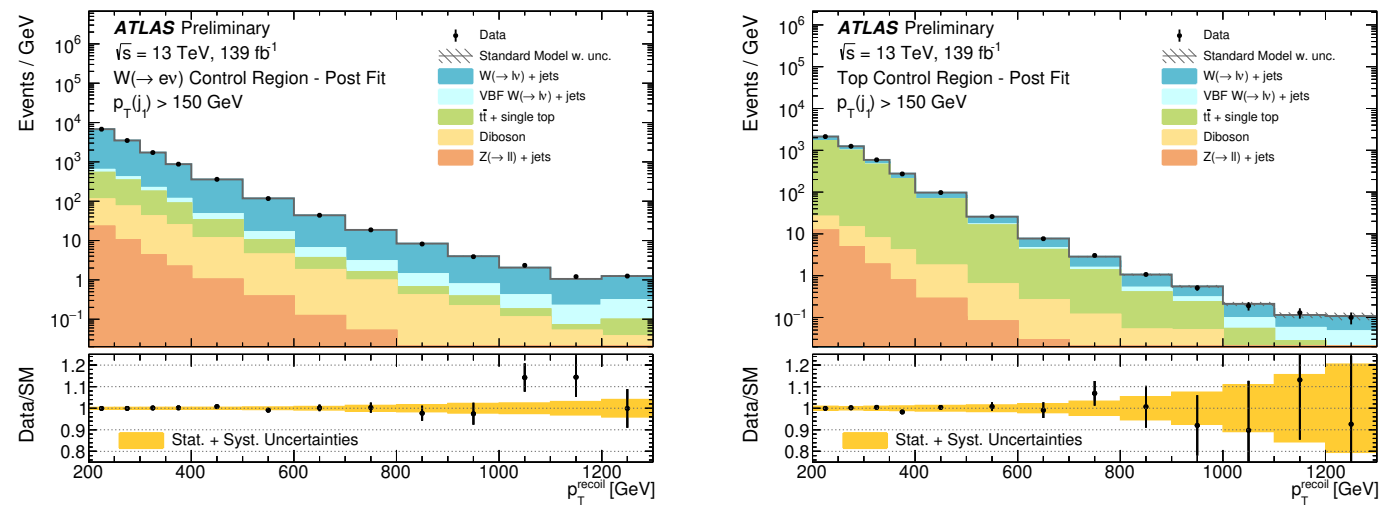

Figure 6: Post-fit plots of the $W(e v)$ and top control regions for the mono-jet analysis. The variable $p_{\mathrm{T}}^{\text {recoil }}$ is the vector sum of the missing transverse momentum $E_{\mathrm{T}}^{\mathrm{miss}}$ and the lepton(s) in each control region. [7]

Background estimation for the mono-jet analysis is done using a data-driven procedure similar to that described in Section 2. One- and two- lepton control regions are defined for $Z+j e t s$ and $W+$ jets background estimation, with an additional control region added for top backgrounds that requires one lepton and one $b$-jet. MC in these regions is reweighted analytically as a function of boson $p_{T}$ to next to next to leading order (NNLO) in QCD, and NLO in electroweak, with Sudakov logarithms at two loops [10]. In addition, the $Z$ and $W$ processes are correlated, meaning that a single transfer factor is used per bin for both weak boson backgrounds. Two additional transfer factors are defined for $t \bar{t}$ and single- $t$ backgrounds.

A simultaneous shape fit was performed, and no significant excess observed. Figure 6 shows post-fit plots in the $W$ and top control regions, and Figure 7 shows a post-fit plot of the signal region. The total uncertainty on the fit is 1.2 to $4.1 \%$ across all bins, and the major sources of systematic uncertainty are electron and muon identification and reconstruction. The very low uncertainty 


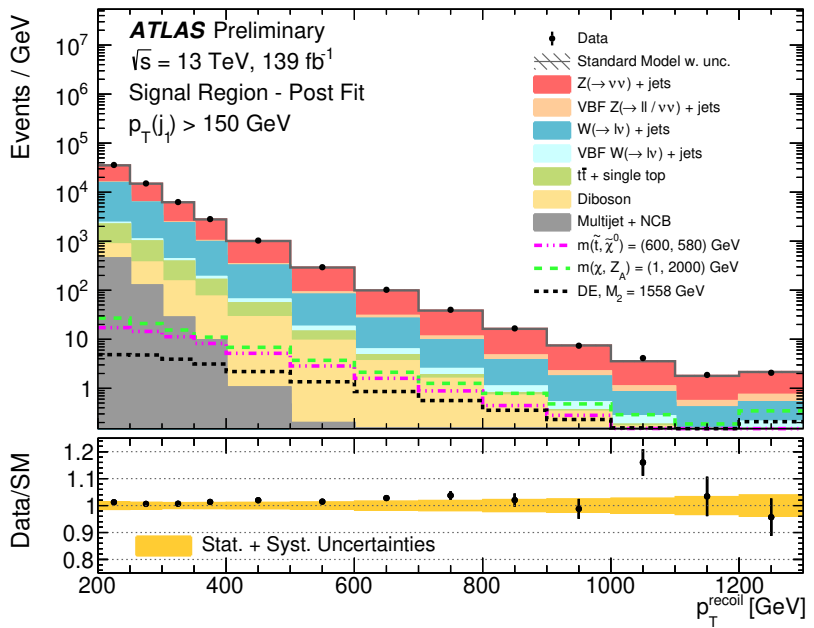

Figure 7: Post-fit plot of the mono-jet analysis signal region. In this plot, the variable $p_{\mathrm{T}}^{\text {recoil }}$ is simply the missing transverse momentum, $E_{\mathrm{T}}^{\text {miss }}$. [7]

enables a limit on $\mathcal{B}_{H \rightarrow \text { inv }}$ to be set, and the $95 \%$ confidence observed (expected) limit is 0.63 (0.57). The $H \rightarrow$ invisible signal acceptance is only $54 \%$ ggF, with $34 \%$ comprised of VBF events and $12 \% \mathrm{VH}$ events, so the analysis is sensitive to multiple invisible production channels.

\section{Conclusion}

Two recent ATLAS results with $139 \mathrm{fb}^{-1}$ of proton-proton collision data that set limits on $\mathcal{B}_{H \rightarrow \text { inv }}$ have been presented. The VBF analysis sets an observed (expected) limit of $0.13\left(0.13_{-0.04}^{+0.05}\right)$, which is significantly improved from the previous ATLAS combination of $0.26\left(0.17_{-0.05}^{+0.07}\right)$ [5]. The mono-jet analysis sets a limit of 0.63(0.57), an interpretation not previously done in run 2 .

\section{References}

[1] ATLas Collaboration, Phys. Lett. B716 (2012) 1 [1207.7214].

[2] CMS Collaboration, Phys. Lett. B716 (2012) 30 [1207.7235].

[3] ATLAS Collaboration, JINST 3 (2008) S08003.

[4] LHC Higgs Cross Section Working Group (Oct, 2016), 10.23731/CYRM-2017-002.

[5] ATlas Collaboration, Phys. Lett. B 793 (2019) 499 [1809. 06682].

[6] ATLAS Collaboration, ATLAS-CONF-2020-008, CERN, Geneva (Apr, 2020).

[7] ATLAS Collaboration, ATLAS-CONF-2020-048, CERN, Geneva (Aug, 2020).

[8] T. Gleisberg, S. Hoeche, F. Krauss et al., JHEP 02 (2009) 007 [0811 . 4622].

[9] G. Arcadi, A. Djouadi and M. Raidal, Phys. Rept. 842 (2020) 1 [1903. 03616].

[10] J.M. Lindert et al., Eur. Phys. J. C77 (2017) 829 [1705 . 04664]. 